\title{
Combined therapy with a thymidylate synthase-inhibiting vector and $S-1$ has effective antitumor activity against 5-FU-resistant tumors
}

\author{
KYUICHI KADOTA ${ }^{1,2}$, CHENG-LONG HUANG ${ }^{1}$, DAGE LIU ${ }^{1}$, HIROYASEU YOKOMISE ${ }^{1}$, \\ REIJI HABA ${ }^{2}$ and HIROMI WADA ${ }^{3}$
}

Departments of ${ }^{1}$ General Thoracic Surgery, Breast and Endocrinological Surgery and ${ }^{2}$ Department of Diagnostic Pathology, Faculty of Medicine, Kagawa University, Kagawa 761-0793; ${ }^{3}$ Department of Thoracic Surgery,

Faculty of Medicine, Kyoto University, Kyoto 606-8507, Japan

Received October 6, 2010; Accepted December 7, 2010

DOI: $10.3892 /$ ijo. 2010.880

\begin{abstract}
High levels of intratumoral thymidylate synthase (TS) expression are associated with resistance to 5-fluorourcil (5-FU). In order to establish a new treatment method for 5-FU-resistant tumors, the efficacy of gene therapy was investigated using an adenoviral vector expressing short hairpin RNA (shRNA) targeting TS. A replication-deficient recombinant adenoviral vector expressing shRNA targeting TS was constructed under the control of the human U6 promoter (Ad-shTS). Three 5-FU-resistant cancer cell lines, DLD-1/5FU, KM12C/5FU and NUGC-3/5FU, were used. Transduction with Ad-shTS effectively downregulated TS expression in all three 5-FU-resistant tumor cells. MTT assays demonstrated that treatment with Ad-shTS significantly inhibited the growth of all three 5-FU-resistant tumor cells. Furthermore, combined treatment with Ad-shTS and 5-FU demonstrated significantly greater inhibition of tumor cell growth in comparison to 5-FU treatment alone and Ad-shTS treatment alone. S-1, a combination of tegafur, gimeracil and oteracil potassium, was used for the 5-FU treatment by in vivo experiments. The combined treatment of Ad-shTS and S-1 was found to have the strongest antitumor effect against 5-FU-resistant DLD-1/5FU xenografts in nude mice in comparison to $\mathrm{S}-1$ treatment alone and Ad-shTS treatment alone. Furthermore, the apoptotic index in tumors treated with combined Ad-shTS and S-1 was significantly higher in comparison to that in tumors treated with S-1 alone and that in tumors treated with Ad-shTS alone. Consequently, the combined treatment of the TS-inhibiting adenoviral vector
\end{abstract}

Correspondence to: Dr Cheng-long Huang, Department of General Thoracic Surgery, Breast and Endocrinological Surgery, Faculty of Medicine, Kagawa University, 1750-1 Miki-cho, Kita-gun, Kagawa 761-0793, Japan

E-mail: chuang@kms.ac.jp

Key words: thymidylate synthase, S-1, 5-FU, shRNA, gene therapy and S-1 has effective antitumor activity against 5-FU-resistant tumors.

\section{Introduction}

Molecular biology studies have revealed that many molecules affect the various biological behaviors of malignant tumors. Therefore, it is important to design an optimal therapeutic strategy depending on the specific tumor biology (1). The selection of an effective chemotherapy regimen based on the evaluation of tumor-associated biomarkers, that is tailormade chemotherapy, can improve the clinical outcome of cancer patients (2). For example, 5-fluorouracil (5-FU)derived agents have widely been used in treatment of various human cancers (3-5). In addition, thymidylate synthase (TS), which plays a role in DNA synthesis (6), is a target molecule of 5-fluorouracil (5-FU) (7). Many clinical studies involving human cancers, including gastrointestinal tumors and nonsmall cell lung cancers (NSCLCs), have shown 5-FU-derived agents may be effective for tumors with a low TS expression (8-10). On the other hand, epidermal growth factor receptor (EGFR)-specific tyrosine kinase inhibitors may be effective for tumors with EGFR gene mutations (11). Therefore, we are utilizing tailor-made chemotherapy based on the coevaluation of TS expression and EGFR gene status for NSCLC patients at present (2). However, because most tumors with a low expression of TS or EGFR mutations are lung adenocarcinomas, a new strategy is required for treatment of the remaining populations of NSCLCs with a high expression of TS and wild-type of EGFR.

Recently, RNA interference (RNAi), a sequence-specific gene silencing process initiated with double-stranded RNA (dsRNA), has been developed to be a powerful tool to inhibit expressions of specific genes $(12,13)$. Considering the fact that a high level of the intratumoral TS expression is associated with 5-FU-resistance and a poor outcome in cancer patients treated with 5-FU-derived agents (8-10), the suppression of TS expression by RNAi might restore the responsiveness to 5-FU in 5-FU-resistant tumors. Furthermore, stable small interfering RNA (siRNA) molecules can be produced in mammalian cells via short hairpin RNA (shRNA), suggesting 
that shRNA-expressing vectors could be usefully employed to obtain a stable siRNA-mediated anti-oncogenic activity $(14,15)$. Therefore, in order to establish an effective treatment for 5-FU-resistant tumors, an adenoviral vector was constructed that expressed shRNA targeting TS. Consequently, the combined treatment with the adenoviral vector expressing shRNA targeting TS and 5-FU has effective antitumor activity against 5-FU-resistant tumor cells in vitro. Finally, the combined treatment with the TS-inhibiting adenoviral vector and an oral 5-FU-derived agent S-1 (a combination of tegafur, gimeracil and oteracil potassium) (16-18) was found to have effective antitumor activity against 5-FU-resistant tumor xenografts.

\section{Materials and methods}

Chemicals. 5-FU and uracil were purchased from Wako Pure Chemical Industries (Osaka, Japan), and S-1 was produced by Taiho Pharmaceutical Co., Ltd. (Tokushima, Japan). All other chemicals used were commercial products of the highest quality available.

Cell lines and cell culture. Three 5-FU-resistant cancer cell lines, including a human colorectal carcinoma DLD-1/5FU (19), a human stomach carcinoma NUGC-3/5FU (20), and a human colorectal carcinoma KM12C/5FU (21), were kindly provided by Taiho Pharmaceutical Co., Ltd. DLD-1/5FU cells and NUGC-3/5FU cells were maintained in RPMI-1640 medium supplemented with $10 \mu \mathrm{M} 5$-FU and $10 \%$ fetal calf serum. KM12C/5FU cells were maintained in modified Eagle's medium supplemented with $10 \mu \mathrm{M}$ 5-FU and $10 \%$ fetal calf serum.

Design and transfection of siRNA. To select the specific siRNA sequence that could effectively suppress the TS expression, three siRNA oligonucleotides targeting TS were designed using the siRNA Design Support System (Takara Biomedicals, Otsu, Japan). The sense strand sequences were as follows: TS-siRNA1, 5'-GUAACACCAUCGAUCAUGATdT-3'; TSSiRNA2, 5'-GAAUACAGAGAUAUGGAAUTdT-3'; and TS-siRNA3, 5'-CGAUCAUGAUGUAGAGUGUTdT-3'. DLD-1/5FU cells were seeded in 6-cm culture dishes $24 \mathrm{~h}$ prior to transfection, to reach $50-60 \%$ confluency. The siRNA transfection was performed in a total volume of $3 \mathrm{ml}$ containing each siRNA (final concentration of $25 \mathrm{nM}$ ) and $25 \mu \mathrm{l}$ of TransIT-TKO transfection reagents (Mirus, Madison WI, USA) according to the manufacturer's instructions.

Construction of adenoviral vectors. After the screening of effective siRNAs targeting TS, the shRNA template [forward strand: 5'-TS-siRNA1 sense strand (GTAACACCATCGAT CATGA) + loop (TAGTGCTCCTGGTTG) + TS-siRNA1 antisense strand (TCATGATCGATGGTGTTAC) + polymerase III terminator (TTTTTT)] was synthesized. In order to produce a plasmid vector expressing shRNA targeting TS (plasmid-shTS), this shRNA template was cloned into a pBAsi-hU6 plasmid vector (Takara Biomedicals), containing a human RNA polymerase III-dependent U6 promoter. All the constructions were confirmed by DNA sequencing. Next, an adenoviral vector was constructed using an Adenovirus Expression vector kit (Takara Biomedicals) according to the manufacturer's protocol. Briefly, the insert with the human U6 promoter and shRNA template was produced from plasmid-shTS using restriction enzyme digestion by EcoRV. The insert was then ligated into a pAxcwit cosmid vector. Using the COS-TPC method (22), an E1-deleted replication-deficient recombinant adenoviral vector expressing shRNA targeting TS was constructed under the control of the human U6 promoter (Ad-shTS). A control adenoviral vector expressing shRNA against the SNC1 sequence (sense sequence: 5'-TCTTAATCGCGTATAA GGC-3', Takara Biomedicals), a negative control siRNA for whole human genes, was also constructed (Ad-shNC). The adenoviral vectors were amplified in HEK293 packaging cells and purified by cesium chloride ultracentrifugation. Functional plaque-forming unit (PFU) titers were determined by limiting dilution plaque titration on HEK293 cells. All of the resulting viral preparations were determined to be free of contamination by wild-type adenovirus and endotoxin.

Real-time quantitative RT-PCR. Total cellular RNA was extracted using the acid guanidinium thiocyanate procedure. First-strand cDNA synthesis was performed with $5 \mu \mathrm{g}$ of total RNA using a cDNA synthesis kit (Amersham Bioscience, Piscataway, NJ, USA). To quantify the $T S$ gene expression, TaqMan real-time quantitative PCR was performed with the ABI PRISM 7700 sequence detection system (Applied Biosystems, Foster City, CA, USA). The primers and probes were from the Assays-on-Demand gene expression assay mix (TS assay ID Hs00426591_m1, PCR product size 87 bp; Applied Biosystems). Each sample was run in triplicate, and each PCR experiment included three non-template control wells. The comparative threshold cycle method (Applied Biosystems) was used to calculate the gene expression in each sample relative to the value observed in control cells, using GAPDH (Assays-on-Demand gene expression system, assay ID Hs99999905_m1, PCR product size 122 bp; Applied Biosystems) as a control for normalization among samples. RNA samples isolated from three independent experiments were evaluated.

Western blot analysis. Cells were harvested and resuspended in lysis buffer (62.5 mM Tris-HCl, 2\% SDS, $10 \%$ glycerol, $4 \mathrm{M}$ urea). Protein samples $(50 \mu \mathrm{g})$ were each diluted into a $20-\mu 1$ solution of lysis buffer and 5\% 2-mercaptoethanol (Bio-Rad Laboratories, Hercules, CA, USA) and heated in a water bath at $95^{\circ} \mathrm{C}$ for $5 \mathrm{~min}$. Then, protein extracts were separated by $10 \%$ SDS-PAGE. Next, the separated proteins were transferred from gel to nitrocellulose membrane (Hybond-ECL; Amersham) and then blocked in a blocking solution (5\% dry milk and $0.2 \%$ Tween-20 in PBS) for $1 \mathrm{~h}$. Then, membranes were incubated overnight with a rabbit polyclonal antibody detecting TS, diluted at 1:500 (1). The membranes were then incubated with HRP-labeled secondary antibodies for $1 \mathrm{~h}$. The proteins were visualized on enhanced chemiluminescence film (Hyperfilm; Amersham) by application of Western blotting detection system (Amersham). Finally, the blots were reprobed using a mouse anti-human actin mAb (C-2, Santa Cruz Biotechnology, Santa Cruz, CA, USA, diluted at 1:1000) to ensure equal loading and transfer 
A

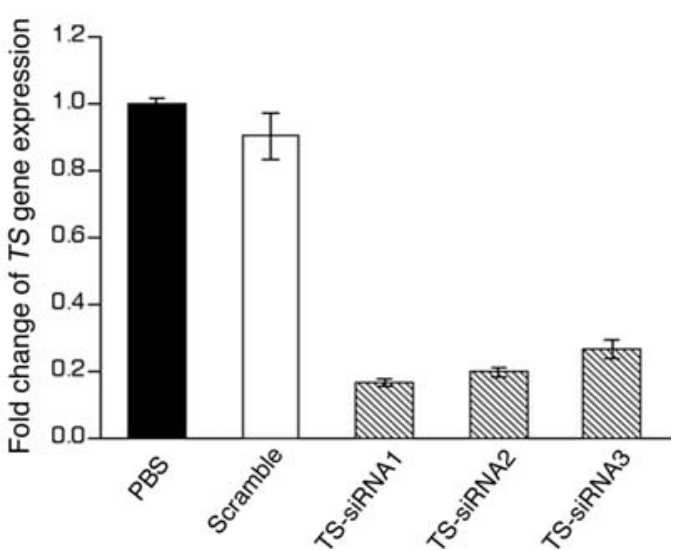

B

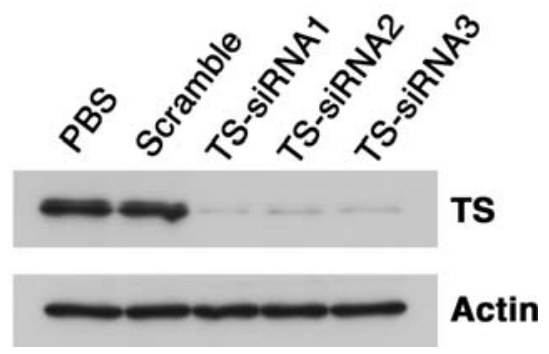

Figure 1. Transfection of siRNAs targeting TS into 5-FU-resistant DLD-1/ 5FU cells. (A) Fold changes of the TS gene expressions evaluated by realtime quantitative RT-PCR; (B) TS protein expressions evaluated by Western blot analyses. One of three experiments with similar results is shown. PBS, a mock control; scramble, a scrambled oligonucleotide as a negative control against TS-siRNA1.

of proteins. Each experiment was repeated three times with consistent results.

Cell viability assay. Tumor cells were seeded in 96-well plates at concentration of 4000 cells/well. At $24 \mathrm{~h}$ after plating, cells were treated with PBS (a mock control), Ad-shTS, Ad-shNC, 5-FU $(0.5 \mu \mathrm{g} / \mathrm{ml} \mathrm{5-FU} \mathrm{and} 30 \mu \mathrm{g} / \mathrm{ml}$ uracil) (23), or the combination of the vector and 5-FU. After $96 \mathrm{~h}$ incubation, the cell viability in each group was determined by 3-(4,5-dimethylthiazol-2-yl)-2,5-diphenyltetrazolium bromide (MTT) assays using a Cell Proliferation Kit I (Roche, Mannheim, Germany). In brief, tumor cells were incubated with $10 \mu \mathrm{l}$ of MTT labeling reagent for $4 \mathrm{~h}$, and then incubated with $100 \mu 1$ of solubilization solution overnight. Finally, the cell viability in each well was measured in terms of optical density at a wavelength of $570 \mathrm{~nm}$, and at $690 \mathrm{~nm}$ for reference. Results were expressed as follows: cell growth inhibition $(\%)=[1$-(the relative percentage of absorbance detected in the treated cells)/(the relative percentage of absorbance detected in the PBS-treated control cells)] x 100 . Each cell viability assay was performed in triplicate and repeated three times.

Tumor xenograft model in nude mice. Regarding the in vivo experiments, S-1 (a combination product of tegafur, gimeracil and oteracil potassium) was used for the 5-FU treatment. A tumor xenograft was prepared by the subcutaneous implantation of approximately $8 \mathrm{~mm}^{3}$ fragments of tumors derived from DLD-1/5FU cells, into the back of six-week-old male nude mice. When the tumor volume reached approximately $100 \mathrm{~mm}^{3}$, the mice were randomly divided into the six groups (six mice/group): control group treated with PBS, groups treated with Ad-shTS, Ad-shNC, S-1, and the combination of the vector and $\mathrm{S}-1$. In groups treated with adenoviral vectors, intratumoral injections of Ad-shTS or Ad-shNC were performed at $2 \times 10^{9}$ PFU every four days for four times. In the groups treated with $\mathrm{S}-1,10 \mathrm{mg} / \mathrm{kg}$ of $\mathrm{S}-1$ was administered orally to nude mice once a day for 14 consecutive days. The first day of the initial treatment was designated as day 1 . Tumor growth was monitored every three days for 30 days by measuring tumor size using a caliper. The tumor volume was calculated according to the following formula: tumor volume $=($ length $) \times(\text { width })^{2} \times 0.5$. All animal experiments were performed in accordance with the Institutional Guide for the care and use of laboratory animals. Animals were euthanized when tumor xenograft exceeded $2 \mathrm{~cm}$ in diameter or at the end point of observations, whichever came first.

Detection of apoptosis. The presence of apoptotic cells was detected with the TUNEL method using the in situ apoptosis detection kit (Takara Biomedicals) according to the manufacturer's protocol. After sections were deparaffinized and rehydrated, the slides were treated for $15 \mathrm{~min}$ with $20 \mu \mathrm{g} / \mathrm{ml}$ proteinase $\mathrm{K}$. After quenching the endogenous peroxidase activity with $3 \% \mathrm{H}_{2} \mathrm{O}_{2}$ for $5 \mathrm{~min}$, the sections were then incubated for $90 \mathrm{~min}$ at $37^{\circ} \mathrm{C}$ with the TUNEL reaction mixture including terminal deoxynucleotidyl transferase (TdT). Next, the sections were incubated for $30 \mathrm{~min}$ at $37^{\circ} \mathrm{C}$ with anti-FITC horseradish peroxidase conjugate. Staining was developed using 3,3'-diaminobenzidine tetrahydrochloride for $15 \mathrm{~min}$. Lastly, the sections were lightly counterstained with Mayer's hematoxylin. Apoptotic cells were determined based on observations of TUNEL-staining sections and serial HE-staining sections. In each case, a total of 10,000 tumor cells were evaluated at high magnification by two authors (K.K. and D.L.) independently. The apoptotic index was defined as the number of apoptotic cells per 1,000 tumor cells.

Statistical analysis. Data are expressed as the mean \pm SD. Differences among the treatment groups were assessed using the Mann-Whitney U-test. $\mathrm{P}<0.05$ was considered to be statistically significant.

\section{Results}

Identification of specific sequence of siRNA targeting TS. First, in order to identify the specific siRNA sequence with optimal inhibitory activity against TS, three chemicallysynthesized siRNAs (TS-siRNA1, TS-siRNA2 and TSsiRNA3) designed from different sequences of the TS mRNA were investigated. Seventy-two hours after the siRNA transfection into 5-FU-resistant colorectal carcinoma DLD-1/5FU cells, the efficiencies of each siRNA on the TS suppression were ascertained in gene expression levels by real-time quantitative RT-PCR and protein expression levels by Western blot analyses. Consequently, the transfection of TS-siRNA1 was found to cause the strongest downregulation of the TS both in gene expression levels $(83.5 \pm 1.3 \%$, Fig. 1A) and 
A

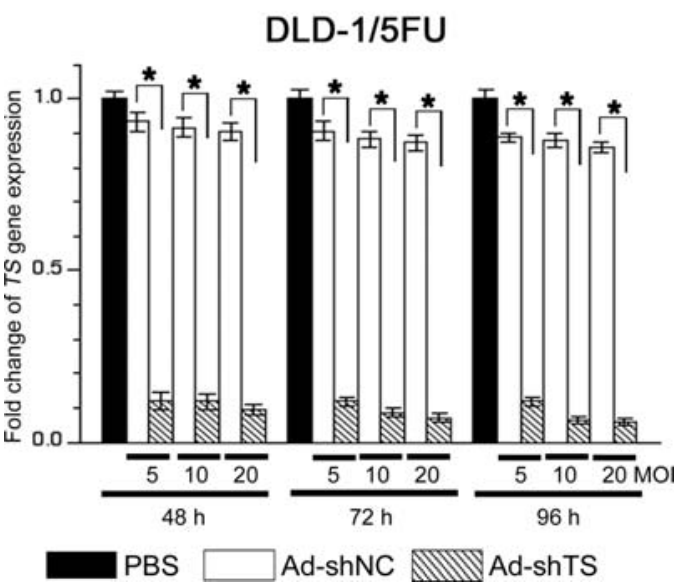

B

\section{DLD-1/5FU}

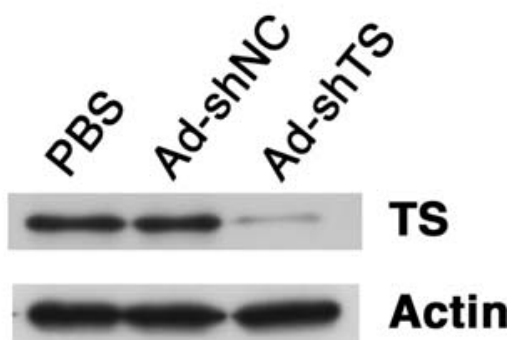

C

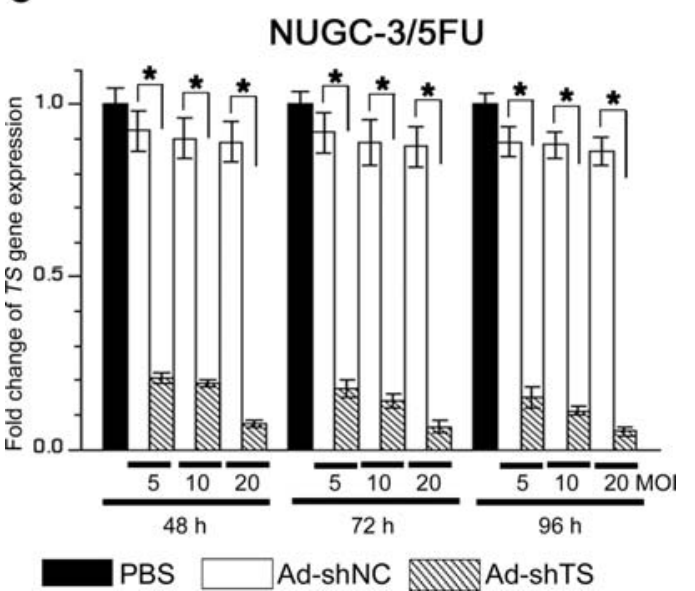

D

\section{NUGC-3/5FU}

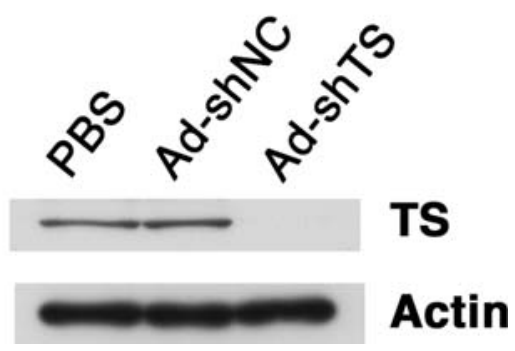

E

$\mathrm{KM} 12 \mathrm{C} / 5 \mathrm{FU}$

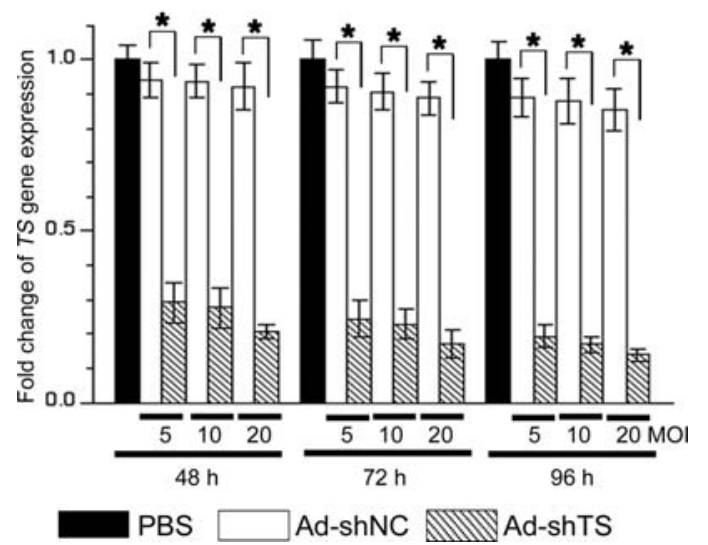

$\mathbf{F}$

\section{$\mathrm{KM} 12 \mathrm{C} / 5 \mathrm{FU}$}

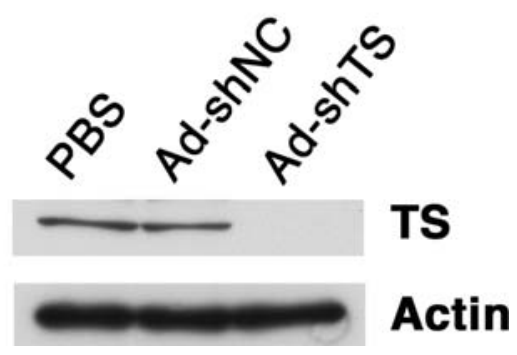

Figure 2. TS gene expressions and TS protein expressions in 5-FU-resistant tumor cell lines after the transduction with adenoviral vector of Ad-shTS or AdshNC (a control vector). Gene expressions (A) and protein expressions (B) in DLD-1/5FU; Gene expressions (C) and protein expressions (D) in NUGC-3/ 5FU; Gene expressions (E) and protein expressions (F) in KM12C/5FU. Regarding protein expressions, Western blot analyses were performed lines at $96 \mathrm{~h}$ after the transduction with adenoviral vector of Ad-shTS or Ad-shNC at a MOI of 20, and one of three experiments with similar results is shown. PBS, a mock control; ${ }^{\mathrm{P}}<0.0001$

protein expression levels (Fig. 1B). As a result, TS-siRNA1 was selected as the specific target site against TS to construct an adenoviral vector expressing shRNA targeting TS.

An adenoviral vector expressing shRNA targeting TS efficiently downregulates the TS expression. In order to investigate the inhibitory effect of the Ad-shTS, three 5-FUresistant human cancer cell lines were transducted with AdshTS or Ad-shNC at a multiplicity of infection (MOI, PFU/ cell) of 5, 10 and 20. Real-time quantitative RT-PCR analyses showed that Ad-shTS effectively knocked down the TS gene expression in all the three 5-FU-resistant tumor cells at 48 , 
A

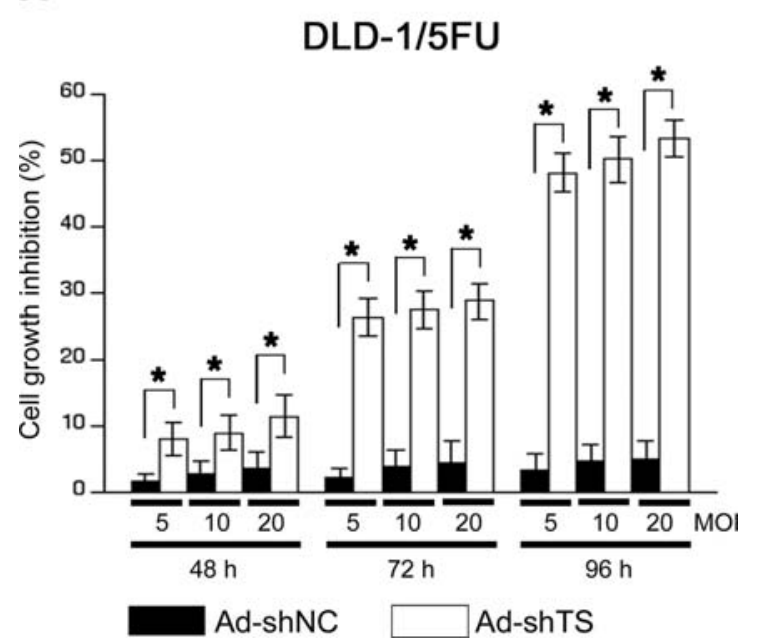

B

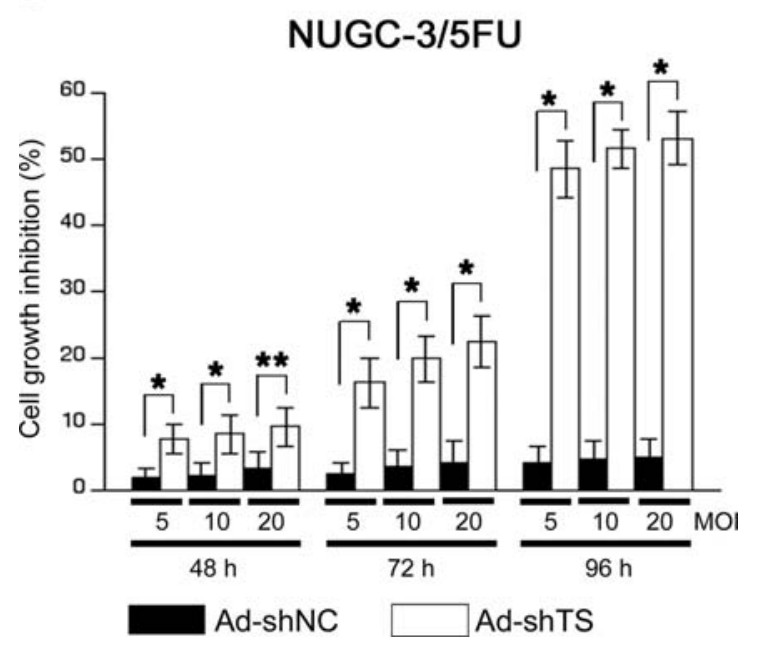

C

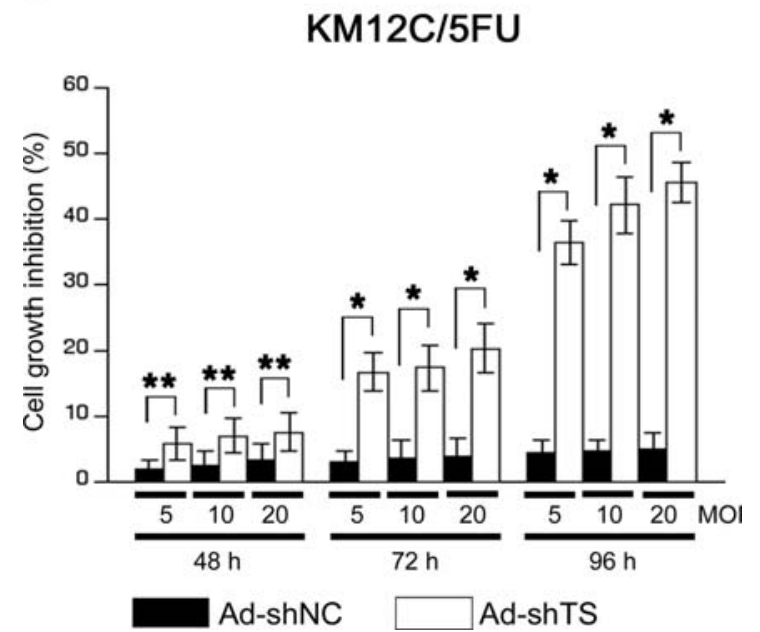

Figure 3. Cell viability evaluated by the MTT assay after the transduction with adenoviral vector of Ad-shTS or Ad-shNC (a control vector). (A) DLD-1/5FU; (B) NUGC-3/5FU; (C) KM12C/5FU. Cell growth inhibition $(\%)=[1$-(the relative percentage of absorbance detected in the treated cells)/(the relative percentage of absorbance detected in the PBS-treated control cells)] x $100 .{ }^{*} \mathrm{P}<0.0001 ;{ }^{* *} \mathrm{P}<0.01$.

72 and $96 \mathrm{~h}$ after transduction (Fig. 2A, C and E). At $96 \mathrm{~h}$ after transduction with Ad-shTS at a MOI of 20, the amount of TS mRNA remaining in cells was $6.0 \pm 0.9 \%$ for DLD-1/ $5 \mathrm{FU}, 5.3 \pm 1.2 \%$ for NUGC-3/5FU, and $13.9 \pm 1.7 \%$ for $\mathrm{KM} 12 \mathrm{C} / 5 \mathrm{FU}$. It was statistically significantly lower than that in cells transducted with Ad-shNC $(\mathrm{P}<0.0001$, respectively). Western blot analyses at $96 \mathrm{~h}$ after transduction also demonstrated that Ad-shTS effectively knocked down the TS protein expression in all the three 5-FU-resistant tumor cells (Fig. 2B, D and F).

An adenoviral vector expressing shRNA targeting TS inhibits growth of 5-FU-resistant tumor cells. Because the TS expression itself is involved in cell proliferation $(24,25)$, we investigated the inhibitory effect of Ad-shTS against the three 5-FU-resistant tumor cell lines. Three 5-FU-resistant human cancer cell lines were transduced with Ad-shTS or Ad-shNC at a MOI of 5,10 and 20. MTT assays were performed at 48, 72 and $96 \mathrm{~h}$ after transduction. Consequently, the simple treatment with Ad-shTS inhibited the growth of all the three 5-FU-resistant tumor cells in a time- and dose-dependent manner. The treatment with Ad-shTS at a MOI of 20 significantly inhibited the growth of all the three 5-FU-resistant tumor cells, by $53.2 \pm 2.8 \%$ for DLD-1/5FU, by $53.0 \pm 4.0 \%$ for NUGC-3/5FU, and by $45.4 \pm 3.0 \%$ for $\mathrm{KM} 12 / 5 \mathrm{FU}$ $(\mathrm{P}<0.0001$ vs. Ad-shNC treatment, respectively) (Fig. 3).

The combined treatment with an adenoviral vector expressing shRNA targeting TS and 5-FU has strong antitumor activity against 5-FU-resistant tumor cells. The TS protein is a target molecule of 5-FU (7), and a high expression of TS is associated with 5-FU-resistance (8-10). Therefore, additional MTT assays were performed to investigate the combined treatment of Ad-shTS and 5-FU against the 5-FU-resistant tumor cells. Three 5-FU-resistant tumor cells were treated with PBS (a mock control), 5-FU $(0.5 \mu \mathrm{g} / \mathrm{ml} \mathrm{5-FU} \mathrm{and} 30 \mu \mathrm{g} / \mathrm{ml}$ uracil) (23), Ad-shTS (20 MOI), Ad-shNC (20 MOI), or the combination of the vector and 5-FU. Cell viability was evaluated by the MTT assay at $96 \mathrm{~h}$ after the treatment. In DLD-1/5FU cells, the growth inhibition rates were $36.7 \pm 4.9 \%$ in 5-FU-treated cells, 53.2 $\pm 2.8 \%$ in Ad-shTS-treated cells, and $77.3 \pm 5.2 \%$ in cells treated with combined Ad-shTS and 5-FU (Fig. 4A). In NUGC-3/5FU cells, the growth inhibition rates were $39.8 \pm 4.1 \%$ in 5 -FU-treated cells, $53.0 \pm 4.0 \%$ in Ad-shTS-treated cells, and $75.0 \pm 3.5 \%$ in cells treated with combined Ad-shTS and 5-FU (Fig. 4B). In KM12C/5FU cells, the growth inhibition rates were $38.5 \pm 3.6 \%$ in $5-\mathrm{FU}$ treated cells, $45.4 \pm 3.0 \%$ in Ad-shTS-treated cells, and $72.3 \pm 4.6 \%$ in cells treated with combined Ad-shTS and 5-FU (Fig. 4C). In all the three 5-FU-resistant tumor cell lines, the combined treatment with Ad-shTS and 5-FU demonstrated a significantly greater inhibition of tumor cell growth in comparison to 5-FU treatment alone ( $\mathrm{P}<0.0001$, respectively) and Ad-shTS treatment alone $(\mathrm{P}<0.0001$, respectively).

The combined treatment with an adenoviral vector expressing shRNA targeting TS and $S-1$ has strong antitumor activity against 5-FU-resistant tumors in vivo. Finally, we investigated the antitumor effect of combined treatment of Ad-shTS and S-1 against 5-FU-resistant tumors in vivo. 5-FU-resistant tumor xenografts in nude mice were prepared by the subcutaneous implantation of tumors derived from 
A

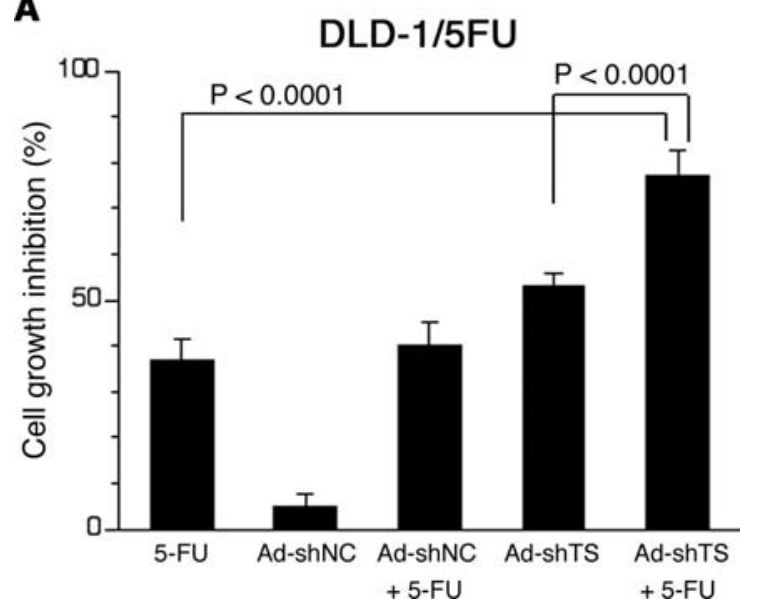

B

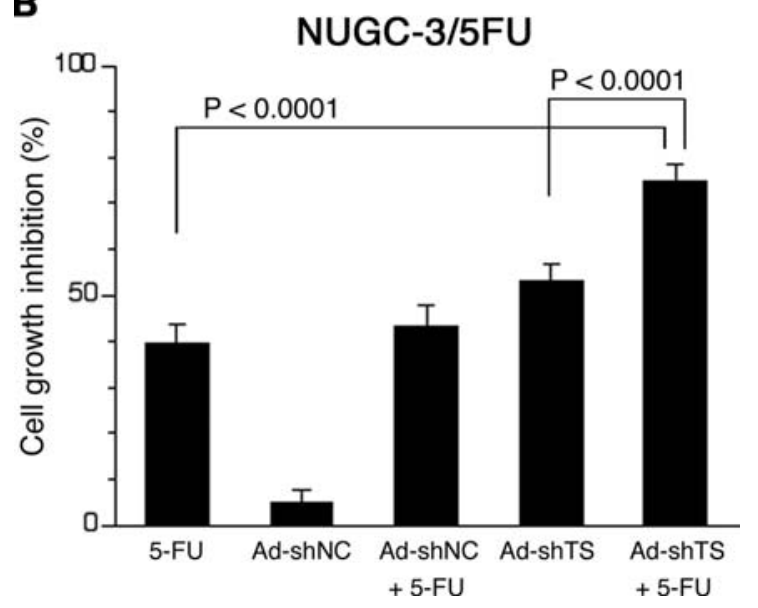

C

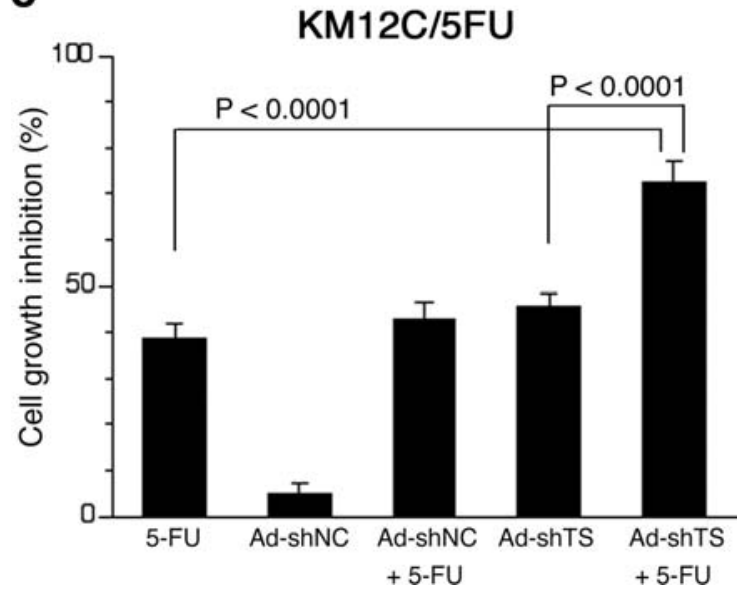

Figure 4. Cell viability evaluated by the MTT assay at $96 \mathrm{~h}$ after the treatment of $5 \mathrm{FU}(0.5 \mu \mathrm{g} / \mathrm{ml} \mathrm{5-FU}$ and $30 \mu \mathrm{g} / \mathrm{ml}$ uracil $)$, Ad-shTS at a MOI of 20, AdshNC (a control vector) at a MOI of 20 , or the combination of the vector and 5-FU. (A) DLD-1/5FU; (B) NUGC-3/5FU; (C) KM12C/5FU. Cell growth inhibition $(\%)=[1-($ the relative percentage of absorbance detected in the treated cells)/(the relative percentage of absorbance detected in the PBStreated control cells)] x 100 .

DLD-1/5FU cells. Mice were randomly divided into the six groups: control group treated with PBS, groups treated with Ad-shTS, Ad-shNC, S-1, and the combination of the vector and S-1 (Fig. 5A). The tumor volume at day 30 was $1655 \pm 264 \mathrm{~mm}^{3}$ in the PBS-treated group, $1294 \pm 306 \mathrm{~mm}^{3}$ in the Ad-shNC-treated group, $929 \pm 139 \mathrm{~mm}^{3}$ in the S-1-treated group, $664 \pm 196 \mathrm{~mm}^{3}$ in the Ad-shTS-treated group, and $212 \pm 15 \mathrm{~mm}^{3}$ in the group treated with combined Ad-shTS and S-1 (Fig. 5B). The Ad-shTS treatment alone had a significantly greater inhibition of DLD-1/5FU xenografts in comparison to PBS treatment and Ad-shNC treatment $(\mathrm{P}<0.01$, respectively). Furthermore, the combined treatment with Ad-shTS and S-1 was found to have a significantly greater inhibition of DLD-1/5FU xenografts in comparison to S-1 treatment alone and Ad-shTS treatment alone $(\mathrm{P}<0.01$, respectively). These results showed that the combined treatment of Ad-shTS and S-1 has the strongest antitumor effect against DLD-1/5FU xenografts.

Furthermore, apoptotic cells in tumor sections obtained at day 30 were analyzed by the TUNEL method (Fig. 6A). The apoptotic indexes were $43.8 \pm 8.7$ in tumors treated with combined Ad-shTS and S-1, 21.1 \pm 5.7 in tumors treated with S-1, 27.9 \pm 7.8 in tumors treated with Ad-shTS (Fig. 6B). The apoptotic index in tumors treated with the combined Ad-shTS and S-1 was significantly higher in comparison to that in tumors treated with $\mathrm{S}-1$ alone and that in tumors treated with Ad-shTS alone $(\mathrm{P}<0.0001$, respectively).

\section{Discussion}

TS catalyses the conversion of deoxypirimidine-5'-monophosphate to deoxythymidine-5'-monophophate. Therefore, TS plays a central role in the biosynthesis of thymidylate, an essential step in DNA synthesis, and its expression is associated with the cell proliferation $(24,25)$. Furthermore, after 5-FU is converted into 5-fluoro-2'-deoxyuridine 5'-monophosphate (FdUMP), this activated molecule forms a tightbinding complex with TS, thus resulting in the inhibition of the TS activity (6). Therefore, TS is a target molecule of 5-FU. In total, the intratumoral TS expression is associated with the cell proliferation $(24,25)$ and the responsiveness to 5-FU (7). In fact, 5-FU-derived agents are considered to be effective for tumors with a low TS expression (2).

In contrast, the overexpression of $\mathrm{TS}$ is reported to be associated with a poor prognosis of many cancer patients treated with 5-FU (8-10). A previous experimental study also reported that E2F1-transfected cells with up-regulation of TS were more resistant to 5-FU (26). Actually, the 5-FUresistant tumor cell lines studied in the present study have high levels of the TS expression (data not shown). Therefore, we considered that the suppression of the intratumoral TS expression could acquire the responsiveness to 5-FU against 5-resistant tumors.

Regarding the method for the downregulation of the target genes, RNAi has recently become a powerful technique for such gene therapy (13). RNAi is a conserved biological process by which dsRNA inhibits gene expression in a sequencedependent manner. This process is initiated by an event by which dsRNA is cleaved into 21- to 23-nucleotide siRNAs by Dicer, a member of the RNase III family. Then, the siRNAs mediates the suppression of the complementary mRNA through a phylogenetically conserved cellular pathway (12).

The successful application of RNAi in cancer gene therapy depends on the efficient delivery of siRNA into cells. Although chemically-synthesized siRNAs are quickly and 
A

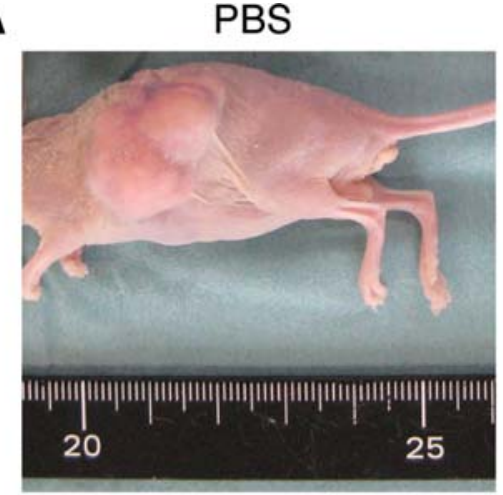

Ad-shNC + S-1

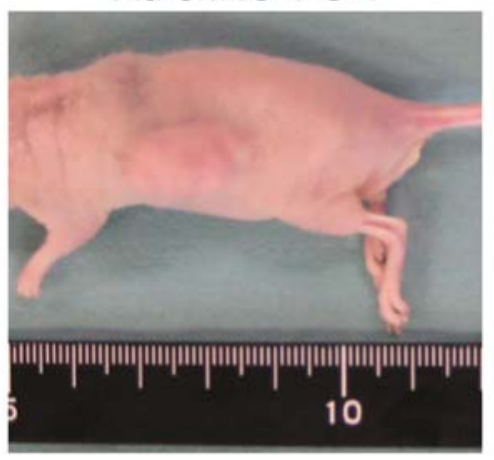

S-1

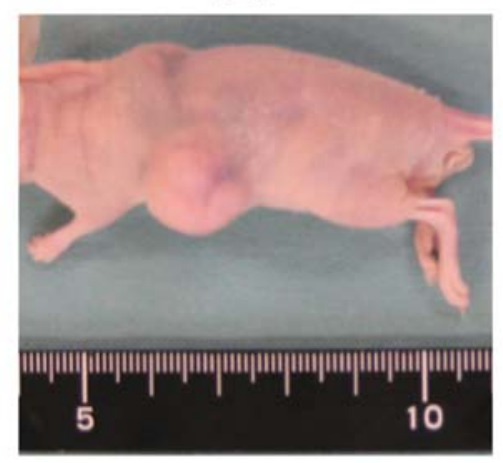

Ad-shTS

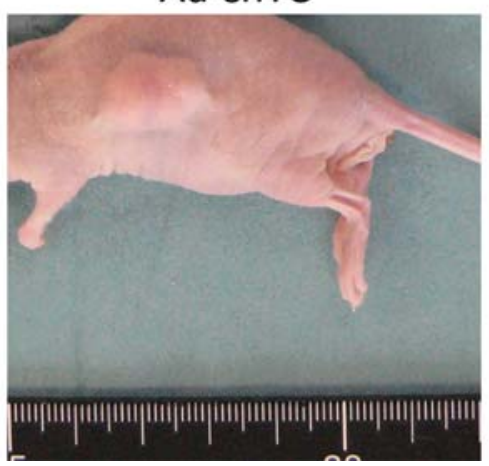

Ad-shNC

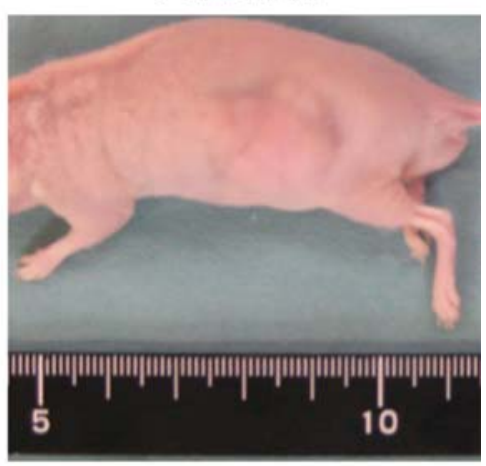

Ad-shTS + S-1

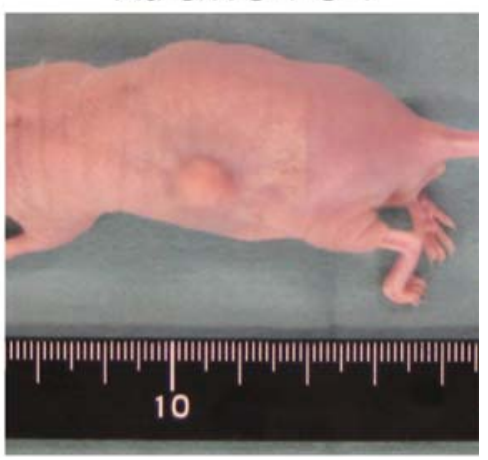

B

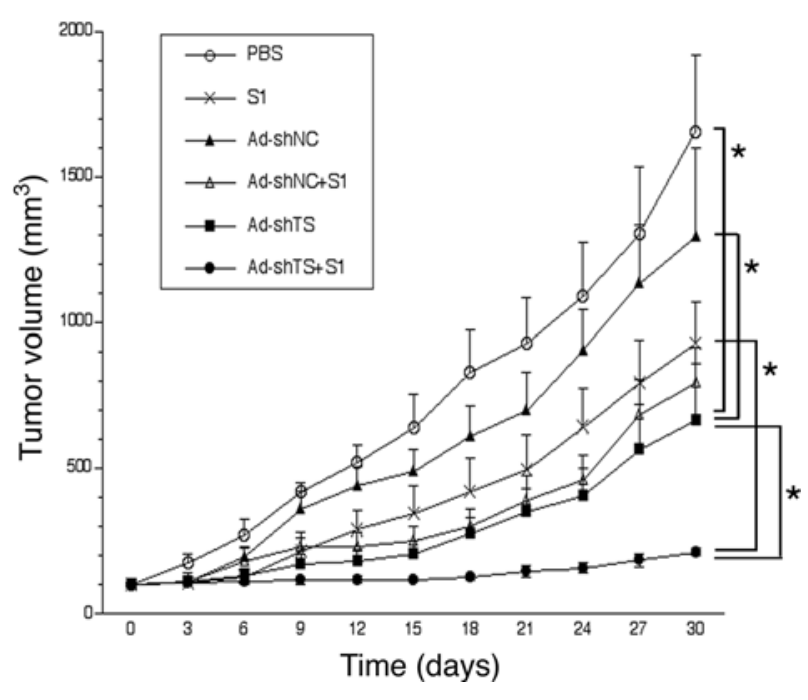

Figure 5. (A) 5-FU-resistant DLD-1/5FU xenografts in nude mice; (B) Tumor volume of DLD-1/5FU xenografts in nude mice. PBS, a mock control; AdshNC, a control vector; ${ }^{*} \mathrm{P}<0.01$.

easily obtained, their application has been restricted because of their short half-lives. In contrast, stable siRNA molecules can be produced in mammalian cells via shRNA expressed under the control of the RNA polymerase III-dependent promoter (14). However, the plasmid-based vectors expressing shRNA perform low transfection efficiency and unstable expression of shRNAs, when used in vivo (27). To circumvent these problems, viral vectors carrying shRNA expression cassettes have been developed in an attempt to achieve high delivery efficacy and long-term expression (15). Among various viral vectors, an adenoviral vector is an efficient tool $(28,29)$. It is relatively easy to produce adenoviral vectors, and high titers of recombinant adenovirus particles can be obtained. Furthermore, as adenoviral vectors can transfect into a broad spectrum of cell types, they have been used widely not only for experimental conditions but also for clinical conditions (30). Therefore, an adenoviral vector expressing shRNA targeting TS was constructed in the present study, and it effectively inhibited the TS expression in human 5-FU-resistant tumor cell lines.

Next, the antitumor effect of this TS-inhibiting adenoviral vector itself against 5-FU-resistant tumor cells was assessed. Consequently, the present study demonstrated that downregulation of the TS expression by simple transduction of the TS-inhibiting adenoviral vector induced apoptosis and inhibited the growth of TS-overexpressing tumor cells, both in vitro and in vivo. Previous research has revealed that TS itself exhibits oncogene-like activity (31). Experimental studies showed that TS expression is associated with tumor cell proliferation $(24,25)$, anchorage-independent growth, and tumor formation in nude mice (31). Furthermore, clinical studies have revealed that a high level of the intratumoral TS expression is associated with tumor proliferation $(1,32)$ and poor prognosis in cancer patients, including gastrointestinal tumors, non-small cell lung cancers, and breast cancers (32-35).

Furthermore, the present study demonstrated that the combined treatment of the TS-inhibiting adenoviral vector and 5-FU more effectively inhibited growth of 5-FU-resistant tumor cells in comparison to TS-inhibiting vector treatment alone and 5-FU treatment alone, not only in vitro but also in vivo. The combined treatment of the TS-inhibiting adenoviral vector and 5-FU strongly induced apoptosis in 5-FU-resistant tumor cell lines, both in vitro and in vivo. These results indicated that the combined therapy with adenoviral vector 
A
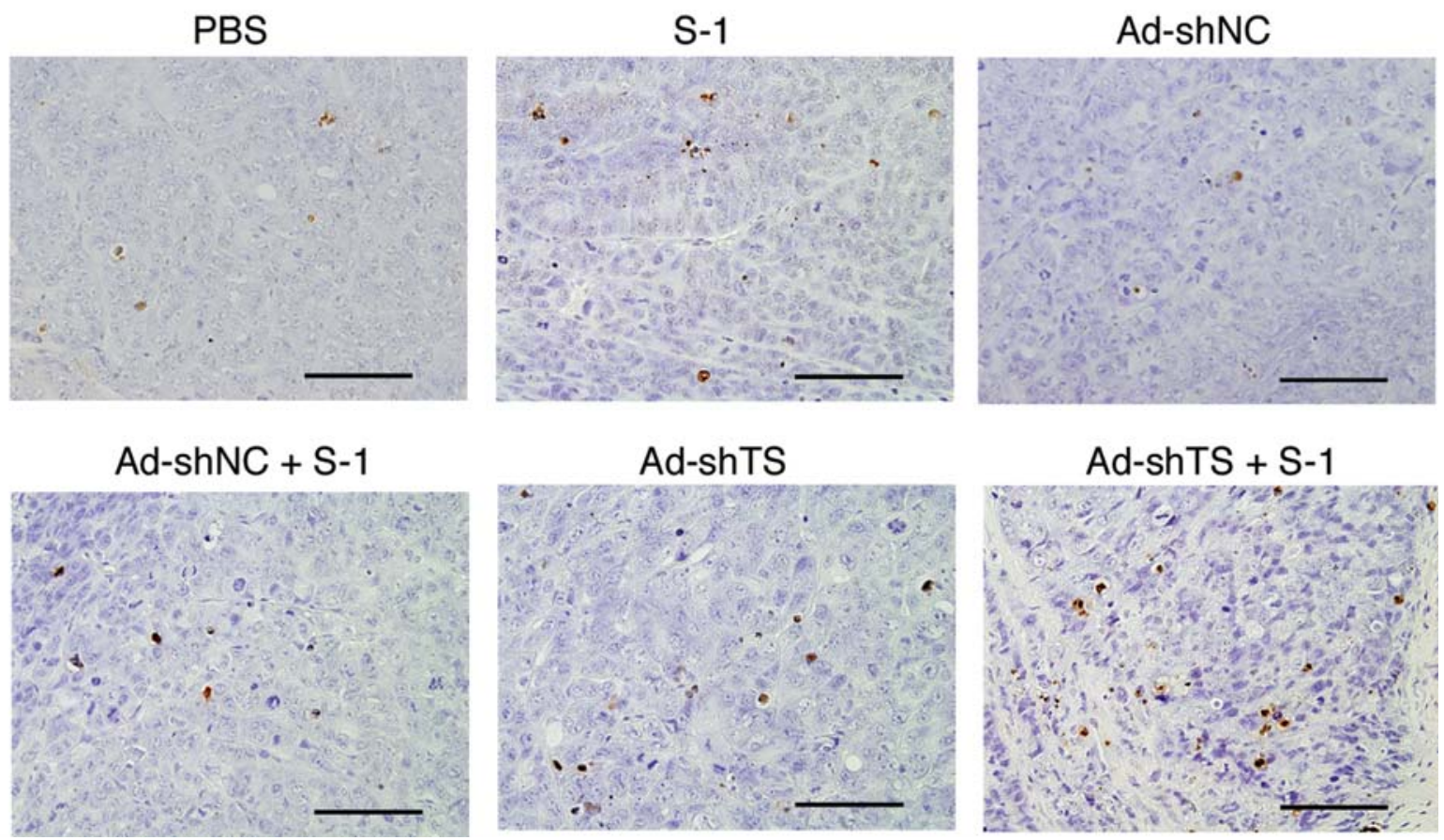

B

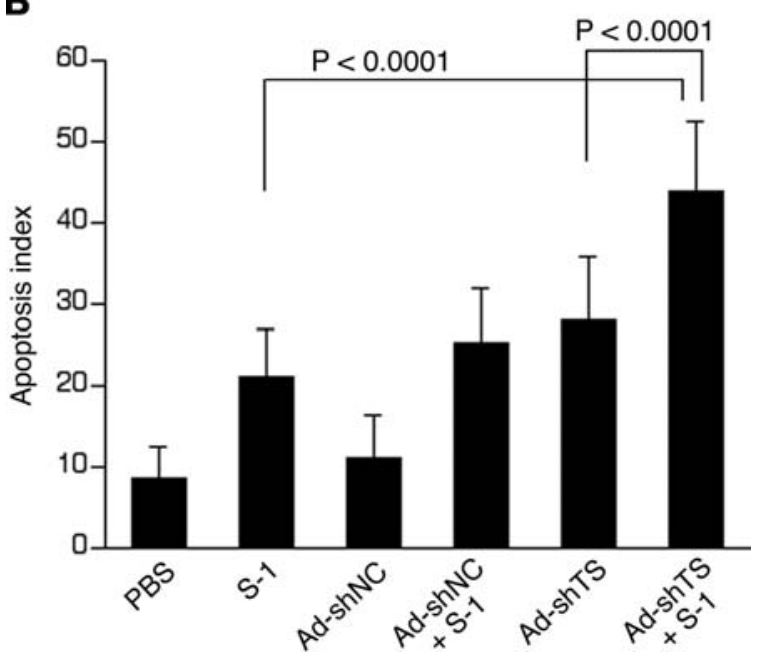

Figure 6. (A) TUNEL staining of 5-FU-resistant DLD-1/5FU xenografts in nude mice at 30 days after the initiation of treatments. Bar, $100 \mu \mathrm{m}$. (B) apoptotic index. PBS, a mock control; Ad-shNC, a control vector.

expressing shRNA targeting TS and S-1 has the strong antitumor activity against 5-FU-resistant tumors. In fact, a previous study also reported the siRNA targeting TS to restore chemosensitivity to TS inhibitor compounds in vitro (36).

Regarding the in vivo experiments, we used S-1 for the 5-FU treatment. Recently, S-1 has been developed for clinical use (16-18). Many experimental and clinical studies have revealed that the responsiveness to 5 -FU is also associated with the intratumoral expression of dihydropyrimidine dehydrogenase (DPD) (8). DPD is the first and rate-limiting enzyme of 5-FU catabolism (37). A high level of the intratumoral DPD expression is thus associated with tumor resistance to 5-FU (8). S-1 is a combination product of tegafur, gimeracil and oteracil potassium. Because gimeracil is a strong inhibitor for the intratumoral DPD activity, we considered that the combined treatment with the TS-inhibiting vector and S-1

could be more effective against 5-FU-resistant xenografts. However, tegafur in S-1 is a prodrug of 5-FU, and it should be converted into 5 -FU by cytochrome p-450 enzyme which is mainly expressed in the liver (3). Therefore, we used 5-FU in in vitro experiments and S-1 in in vivo experiments in the present study.

Consequently, the present study demonstrated the combined treatment with the TS-inhibiting adenoviral vector and S-1 to be successfully used for 5-FU-resistant xenografts. Therefore, this combined treatment could be a new and effective treatment option against 5-FU-resistant tumors. Because the efficacy of an RNAi cancer therapeutics is limited by the quantity of the oligomer that effectively enters the tumor cells, this is primarily dependent on the method of delivery in the clinical setting (15). Further studies including non-viral vectors may be required to develop this new treatment strategy for patients with 5-FU-resistant tumors (38).

The mechanisms of resistance to chemotherapy partly depend on overexpression of the target molecules of antitumor drugs. For example, RRM1 overexpression is associated with resistance to gemcitabine (39). Considering the results of the present study, gene therapy inhibiting these target molecules associated with antitumor drugs could thus enhance the effect of chemotherapy against chemo-resistant tumors. Therefore, combined treatment with the antitumor drug and the target molecule-inhibiting gene therapy will be a new strategy for cancer therapy.

\section{Acknowledgements}

We thank Dr Masakazu Fukushima (Taiho Pharmaceutical Co., Ltd.) for his technical assistance. This study was supported by Grants-in-Aid for Scientific Research from the Japanese Society for the Promotion of Science (no. 18390379). (C. Huang). 


\section{References}

1. Huang C, Liu D, Masuya D, et al: Clinical application of biological markers for treatments of resectable non-small-cell lung cancers. Br J Cancer 92: 1231-1239, 2005.

2. Huang C, Yokomise H, Fukushima M and Kinoshita M: Tailormade chemotherapy for non-small cell lung cancer patients. Future Oncol 2: 289-299, 2006.

3. Takiuchi $\mathrm{H}$ and Ajani JA: Uracil-tegafur in gastric carcinoma: a comprehensive review. J Clin Oncol 16: 2877-2885, 1998.

4. Macdonald JS and Astrow AB: Adjuvant therapy of colon cancer. Semin Oncol 28: 30-40, 2001.

5. Wada H, Hitomi S and Teramatsu T: Adjuvant chemotherapy after complete resection in non-small-cell lung cancer. West Japan study group for lung cancer surgery. J Clin Oncol 14: 1048-1054, 1996.

6. Rustum YM, Harstrick A, Cao S, et al: Thymidylate synthase inhibitors in cancer therapy: direct and indirect inhibitors. J Clin Oncol 15: 389-400, 1997.

7. Beck A, Etienne MC, Cheradame S, et al: A role for dihydropyrimidine dehydrogenase and thymidylate synthase in tumour sensitivity to fluorouracil. Eur J Cancer 30A: 1517-1522, 1994.

8. Nakano J, Huang C, Liu D, et al: Evaluations of biomarkers associated with 5-FU sensitivity for non-small-cell lung cancer patients postoperatively treated with UFT. Br J Cancer 95: 607-615, 2006.

9. Johnston PG, Lenz HJ, Leichman CG, et al: Thymidylate synthase gene and protein expression correlate and are associated with response to 5-fluorouracil in human colorectal and gastric tumors. Cancer Res 55: 1407-1412, 1995.

10. Yeh KH, Shun CT, Chen CL, et al: High expression of thymidylate synthase is associated with the drug resistance of gastric carcinoma to high dose 5-fluorouracil-based systemic chemotherapy. Cancer 82: 1626-1631, 1998.

11. Mitsudomi T, Kosaka T, Endoh H, et al: Mutations of the epidermal growth factor receptor gene predict prolonged survival after gefitinib treatment in patients with non-small-cell lung cancer with postoperative recurrence. J Clin Oncol 23: 2513-2520, 2005.

12. Hannon GJ: RNA interference. Nature 418: 244-251, 2002.

13. Huang C, Li M, Chen C and Yao Q: Small interfering RNA therapy in cancer: mechanism, potential targets, and clinical applications. Expert Opin Ther Targets 12: 637-645, 2008.

14. Brummelkamp TR, Bernards R and Agami R: A system for stable expression of short interfering RNAs in mammalian cells. Science 296: 550-553, 2002.

15. Rao DD, Vorhies JS, Senzer N and Nemunaitis J: siRNA vs. shRNA: similarities and differences. Adv Drug Deliv Rev 61: 746-759, 2009

16. Boku N, Yamamoto S, Fukuda H, et al: Fluorouracil versus combination of irinotecan plus cisplatin versus S-1 in metastatic gastric cancer: a randomised phase 3 study. Lancet Oncol 10 1063-1069, 2009.

17. Yoshioka T, Kato S, Gamoh M, et al: Phase I/II study of sequential therapy with irinotecan and S-1 for metastatic colorectal cancer. Br J Cancer 101: 1972-1977, 2009.

18. Ohyanagi F, Yamamoto N, Horiike A, et al: Phase II trial of S-1 and cisplatin with concurrent radiotherapy for locally advanced non-small-cell lung cancer. Br J Cancer 101: 225-231, 2009.

19. Murakami Y, Kazuno H, Emura T, Tsujimoto H, Suzuki N and Fukushima M: Different mechanisms of acquired resistance to fluorinated pyrimidines in human colorectal cancer cells. Int J Oncol 17: 277-283, 2000

20. Inaba M, Mitsuhashi J, Sawada H, et al: Reduced activity of anabolizing enzymes in 5-fluorouracil-resistant human stomach cancer cells. Jpn J Cancer Res 87: 212-220, 1996.
21. Fukushima M, Fujioka A, Uchida J, Nakagawa F and Takechi T: Thymidylate synthase (TS) and ribonucleotide reductase (RNR) may be involved in acquired resistance to 5-fluorouracil (5-FU) in human cancer xenografts in vivo. Eur J Cancer 37: 1681-1687, 2001.

22. Kanegae Y, Makimura M and Saito I: A simple and efficient method for purification of infectious recombinant adenovirus. Jpn J Med Sci Biol 47: 157-166, 1994.

23. Takechi T, Uchida J, Fujioka A and Fukushima M: Enhancing 5-fluorouracil cytotoxicity by inhibiting dihydropyrimidine dehydrogenase activity with uracil in human tumor cells. Int J Oncol 11: 1041-1044, 1997.

24. Navalgund LG, Rossana C, Muench, AJ and Johnson LF: Cell cycle regulation of thymidylate synthetase gene expression in cultured mouse fibroblasts. J Biol Chem 255: 7386-7390, 1980.

25. Derenzini M, Montanaro L, Trere D, et al: Thymidylate synthase protein expression and activity are related to the cell proliferation rate in human cancer cell lines. Mol Pathol 55: 310-314, 2002.

26. Banerjee D, Schnieders B, Fu JZ, Adhikari D, Zhao SC and Bertino JR: Role of E2F-1 in chemosensitivity. Cancer Res 58: 4292-4296, 1998.

27. Sui G, Soohoo C, Affar el B, et al: A DNA vector-based RNAi technology to suppress gene expression in mammalian cells. Proc Natl Acad Sci USA 99: 5515-5520, 2002.

28. Anderson WF: Human gene therapy. Nature 392: 25-30, 1998.

29. Vile R: Cancer gene therapy-new approaches to tumour cell killing. J Gene Med 2: 141-143, 2000.

30. Keedy V, Wang W, Schiller J, et al: Phase I study of adenovirus p53 administered by bronchoalveolar lavage in patients with bronchioalveolar cell lung carcinoma: ECOG 6597. J Clin Oncol 26: 4166-4171, 2008.

31. Rahman L, Voeller D, Rahman M, et al: Thymidylate synthase as an oncogene: a novel role for an essential DNA synthesis enzyme. Cancer Cell 5: 341-351, 2004.

32. Hashimoto H, Ozeki Y, Sato M, et al: Significance of thymidylate synthase gene expression level in patients with adenocarcinoma of the lung. Cancer 106: 1595-1601, 2006.

33. Tsujitani S, Konishi I, Suzuki K, et al: Expression of thymidylate synthase in relation to survival and chemosensitivity in gastric cancer patients. J Exp Clin Cancer Res 19: 189-195, 2000.

34. Edler D, Kressner U, Ragnhammar P, et al: Immunohistochemically detected thymidylate synthase in colorectal cancer: an independent prognostic factor of survival. Clin Cancer Res 6: 488-492, 2000.

35. Romain S, Spyratos F, Descotes F, et al: Prognostic of DNAsynthesizing enzyme activities (thymidine kinase and thymidylate synathase) in 908 T1-T2, N0-N1, M0 breast cancers: a retrospective multicenter study. Int J Cancer 87: 860-868, 2000.

36. Schmitz JC, Chen T and Chu E: Small interfering double-stranded RNAs as therapeutic molecules to restore chemosensitivity to thymidylate synthase inhibitor compounds. Cancer Res 64: 1431-1435, 2004 .

37. Fischel JL, Etienne MC, Spector T, Formento P, Renee N and Milano G: Dihydropyrimidine dehydrogenase: a tumoral target for fluorouracil modulation. Clin Cancer Res 1: 991-996, 1995.

38. Ma B, Zhang S, Jiang H, Zhao B and Lv H: Lipoplex morphologies and their influences on transfection efficiency in gene delivery. J Control Release 123: 184-194, 2007.

39. Rosell R, Danenberg KD, Alberola V, et al: Ribonucleotide reductase messenger RNA expression and survival in gemcitabine/ cisplatin-treated advanced non-small cell lung cancer patients. Clin Cancer Res 10: 1318-1325, 2004. 